\title{
Clinical challenges of craniopharyngioma: Neurobehavioral sequelae and complex outcomes
}

\author{
Gordon J. Horn ${ }^{* 1}$, Frank D. Lewis ${ }^{2}$ and Joseph Pepitone ${ }^{3}$ \\ ${ }^{1}$ NeuroRestorative Research Institute, Florida State University, College of Medicine Orlando, Florida USA \\ ${ }^{2}$ NeuroRestorative Research Institute Medical College of Georgia at Augusta University Augusta, GA USA \\ ${ }^{3}$ Florida State University, College of Medicine Orlando, Florida USA
}

\begin{abstract}
Craniopharyngioma is a benign tumor with solid or mixed solid-cystic epithelial material found within the pituitary stalk hollow. These tumors have the capacity to impact hormonal regulation of hunger and thirst, kidney function, thyroid regulation, and adrenal gland management. It can occur at any age but most commonly presents in childhood from 5-14 year of age, with a second peak incidence in middle adulthood. Too often Patients with these tumors are often misdiagnosed until the size of the tumor aggressively infiltrates the region producing medical to neurological dysregulation. The current case highlights the complexity of measured outcomes with childhood onset. The case is further compared to two clinical samples for demonstration of impairment. Unfortunately, the damage caused from these tumors is life long and often requires significant supports and services. The goal is establishing and maintaining medical, neurological, and neurobehavioral stability with a multidisciplinary neurorehabilitation level of care.
\end{abstract}

\section{Craniopharyngioma neuropathology and incidence rates}

A craniopharyngioma is a benign, parasellar tumor with solid or mixed solid-cystic epithelial material found within the pituitary stalk hollow [1]. More specifically, it is rare (1-3\% of all brain tumors) and formed from remnants of the embryologic structure of Rathke's Pouch, along a line from the nasopharynx to the diencephalon. These tumors have the capacity to impact hormonal regulation of hunger and thirst, kidney function, thyroid regulation, and adrenal gland management [2]. It can occur at any age but most commonly presents in childhood or adolescence. Patients with these tumors are often misdiagnosed initially until the effects from the size of tumor becomes aggressive to cause a variety of symptoms ranging from medical to neurological dysregulation. The skull based procedures for tumor removal show good long-term tumor control rates [3]. However, retrochiasmatic tumor location, recurrence, and additional surgery were associated with poorer outcomes [4].

The World Health Organization standardized incidence rate is 1.86 (1.60-2.14) for all ages and 2.14 (1.53-2.92) for children (age $<15$ years), with peak incidence rates observed in age groups 5-9 and 40-44 years [5]. Additional research corroborated the first peak incidence between 5 and 14 years of age, but suggested a second peak later for adults falling between 50 and 75 years of age [2]. Regardless, the presenting age can affect the long-term sequelae of patient outcomes. Patients with childhood-onset craniopharyngioma experienced significantly more long-term impairment including growth hormone deficiency, diabetes insipidus, panhypopituitarism, morbid obesity, epilepsy, and psychiatric conditions compared with patients who experienced an adult-onset of craniopharyngioma. Physically, $98 \%$ of craniopharyngioma survivors had pituitary hormone deficiencies, $75 \%$ had visual disturbances, and $56 \%$ were obese [6].
In this particular population, the spectrum of conditions one could develop secondary to tumor removal is largely dependent upon the precise location of the craniopharyngioma, and its relationship to adjacent structures. While the mechanism is not entirely understood, there is a wide variation in the behavioral changes noted post-resection, some of which include attention, somatic, and socializing difficulties [4].

Often this tumor type is described by medical and by surgical outcomes. Surgical outcome is typically defined by excision of the tumor. Medical outcome is based on medical stability of laboratory findings following surgical resection. However, there are often neurobehavioral consequences that occur as a result from the location and resection on the pituitary. The purpose of this case report is to provide insight into the complexity of the condition, complexity of presentation, and demonstrate insights for long-term functional outcome expectations based on neurocognitive and neurobehavioral components of recovery.

\section{The single case report}

The case report provides the following history of the individual studied while providing services with the outcome goal of reduced disability. In addition to the individual, it is important to have various benchmarks to understand the nature of severity and complexity of presentation. Therefore, comparisons are provided with similar clinical groups to demonstrate the impact of this condition and provide insight

Correspondence to: Gordon J. Horn, Ph.D. 341 N. Maitland Avenue Suite 135 Maitland, FL 32751, USA; Tel: (727) 647-0697, E-mail: gordon.horn@ neurorestorative.com

Key words: case study, craniopharyngioma, neurobehavioral outcomes, neurorehabilitation

Received: March 27, 2017; Accepted: April 26, 2017; Published: April 29, 2017 
as to why a person may remain disabled life-long despite the tumor being successfully extracted.

\section{Case history}

The subject is a 28 -year old gentleman with a complex resection of a craniopharyngioma at the age of 15 with chronic medical, neurological, and neurobehavioral impairment. Diagnosis and resection were provided within the first age peak described in the incidence data. The subject has been disabled from productive activity since the surgical resection, though was able to complete formal education at the high school level with educational supports. As an adult, the subject has not been able to work. He has been unable to live at home, or have a relationship, due to significant behavioral disruptions including agitation, irritability, aggression, substance abuse, and legal entanglements. In addition, he has been admitted and discharged from various neurorehabilitation and neurobehavioral programs due to physical aggression and inability to consistently use social and behavioral control in the community.

In addition to the primary diagnosis, secondary related diagnoses included hematuria, vitamin D deficiency, constipation, candidiasis, dysuria, diabetes insipidus, insomnia, seizure disorder, bladder dyscontrol, panhypopituitarism, obesity (ranging from 310 to $400+$ lbs.), non-alcoholic steatohepatitis, organic brain syndrome with cognitive and neurobehavioral impairment, and fluctuating mood disorder with mixed emotional features secondary to his general medical condition. Medications being prescribed at the time of this review included: Depakote ER; Desmopression; Lexapro; Ranitidine; Concerta; Topiramate; Levothyroxine; Prednisone; Hydroxine; Naltrexone; and Calcium Carbonate. Additional medication as needed included Vistaril; Geodon; Epi-Pen; and Lorazepam. All of the medication prescribed fell within the standard ranges of dosing. Medication has continuously changed since the time of resection based on medical, neurological, and psychiatric presentation.

\section{Rehabilitation treatment}

The case study has been provided residential neurobehavioral programming with daily behavioral analysis, planning, and intervention with reinforcement of positive and prosocial behaviors, and extinction of negative behaviors. In addition, the subject has been receiving physical therapy, occupational therapy, speech therapy, recreational programming that is community focused, counseling, case management, and medical management provided by nursing, primary care, and neuropsychiatry.

\section{Measurement of disability}

The Mayo Portland Adaptability Inventory-4 (MPAI-4) was used to determine the functional level of disability for the single case, and for the clinical groups being used as benchmark comparisons. This measure provides construct (e.g., level of disability) validity within various post-hospital brain injury rehabilitation samples. The MPAI-4 has undergone rigorous psychometric testing and has proven reliability and validity as determined through Rasch analysis, Item Cluster, Principle Component Analyses (PCA), and measures of concurrent and predictive validity [7]. Of particular importance, Rasch analysis conducted with the MPAI-4 has revealed strong item reliability that demonstrates the independence of the MPAI-4 individual items, which allows for item comparison among groups or individuals. In addition, item and person reliability, along with person separation and internal consistency of the scale have been well documented, making this an acceptable measurement instrument to identify individual differences uniquely contributing to functional outcome $[8,9]$.

The MPAI-4 has three impairment indices (Abilities, Adjustment, and Participation) derived from 29 items measuring various aspects of disability [8]. The Abilities Index addresses the impact of physical, communication, and cognitive items. The Adjustment Index provides an indication of the neurobehavioral impact on disability. The items include measurement of anxiety, depression, agitation-irritabilityaggression, social awareness, social appropriateness, pain, fatigue, symptom sensitivity, and family relations. The Participation Index provides measurement of applied skills such as initiation, social contact, self-care, home skills, productivity, and money management.

\section{Demographics comparisons with clinical groups}

The subject's disability was measured in two time periods to demonstrate functional outcome based on complex neurobehavioral treatments, and was compared with two clinical groups to determine the level of severity and disability characteristics. The clinical groups were refined from a larger database of 2,606 persons who were admitted to and discharged from multiple programs within 23 states across the United States. Patients in this database had their admission and discharge disability ratings determined by treatment team consensus using the Mayo Portland Adaptability Inventory-4. Using this database, two clinical groups were developed to match our patient in demographic and clinical characteristics; this was for the purpose of generating optimally comparable clinical courses. Each group included those individuals who met the following criteria: admission to neurorehabilitation programs with neurobehavioral management [10]; individuals with admission and discharge assessments; chronicity (i.e., measurement of time from injury to rehabilitation admission) of $48+$ months post-injury; average length of stay greater than 1 year in rehabilitation for stability of program intervention; and male patients only with an age range of 20-40 years. See Tables 1 and 2 for demographic comparisons of the clinical groups.

The first comparison sample $(n=41)$ represents a mix of neurobehaviorally involved brain injury patients with complex medical conditions that interfere with neurological functions such as seizure disorder, metabolic disease, and brain tumors. This sample best represents the complexity and the diversity of this individual's presentation because he has mixed medical, neurological and neurobehavioral sequelae. The second group $(n=30)$ is a chronic traumatic brain injury sample with neurobehavioral impairments

Table 1. Chronic traumatic brain injured group.

\begin{tabular}{|c|c|}
\hline Sample Size & 30 \\
\hline Chronicity & Mean $=88.98$ months, SD $=43.69$ months \\
\hline Length of stay & Mean $=25.03$ months, SD $=12.19$ months \\
\hline Gender & $100 \%$ male \\
\hline Age & Mean $=31.10, \quad$ SD 6.1, range $20-40$ years \\
\hline Program Type & Neurorehabilitation/Neurobehavioral \\
\hline
\end{tabular}

Table 2. Chronic mixed neurologic group.

\begin{tabular}{|c|c|}
\hline Sample Size & 41 \\
\hline Chronicity & Mean $=183.91$ months, SD $=99.43$ months \\
\hline Length of stay & Mean $=14.16$ months, SD $=14.01$ months \\
\hline Gender & $100 \%$ male \\
\hline Age & $\begin{array}{c}\text { Mean }=26.50 \text { years, SD 5.6, } \\
\text { range } 20-40 \text { years }\end{array}$ \\
\hline Program Type & Neurobehavioral \\
\hline
\end{tabular}


without the conditions of the first clinical group. The second group was used to establish the magnitude of disability for a well-researched neurologically impaired matched group. Both groups were similar in presentation based on MPAI-4 scores due to the chronicity falling $>4$ years post-injury, and provide benchmarks for admissions and discharge disability ratings. This, thereby, provides a benchmark for comparing the case with a comparably injured mixed diagnosis group.

To further understand the clinical groups, multivariate statistical analysis was performed to determine if differences existed because of group affiliation. A repeated measures split-plot MANOVA revealed a significant main effect for pre-post testing for both groups, F $(1,69)$ $=58.88, \mathrm{p}<.0005$, Wilks' Lambda $=.54$, partial eta ${ }^{2}=.46$, power to detect $=1.00$. This result demonstrates that the type of multidisciplinary intervention provided for these two groups reduced subjects level of disability at the time of treatment discharge. The individual subject has been receiving the same treatment as the two clinical groups, and therefore, in time, the treatment would be expected to provide similar efficacy in reducing his disability.

In addition, mean differences from admission to discharge between the two groups were not statistically significant, $\mathrm{p}<.149$, N.S. This finding demonstrates that treatment is effective for both groups, and neither group based on the selection criteria, showed a greater advantage toward improved functional outcome based on the therapeutic milieu within these types of centers. See Table 3 for details of the scores from admission to discharge for each clinical group.

With inclusion criteria establishing comparability of the two groups on demographic variables, we were then able to examine the impact of the diagnostic condition of the subject on outcomes. This analysis allowed for comparison of the case subject on all 29 items of the MPAI-4 with the mixed neurologic sample. The mixed sample was only used in this analysis due to the similarity of diagnosis with the case subject. The overall result revealed that the subject's disability rating was higher than the mixed neurologic clinical sample on virtually all of the MPAI- 4 items. In addition, each of the 29 items revealed a complex presentation of impairment across physical, cognitive, communication, neurobehavioral, and instrumental activities of daily living skills, which was consistent with prior research prediction.

\section{Treatment impact}

The case subject has been receiving combined multidisciplinary neurorehabilitation and neurobehavioral treatment as described above. Graphs 1, 2, and 3 provide a comparison of the individual subject compared to the mixed clinical sample only since this sample best represents the broad complexity of impairment. In each of the following graphs, the green line represents the subject after 2 years of intervention, and the blue and red lines are the national mixed sample used for comparison at admission and discharge, respectively.

Abilities Index Graph 1: This analysis shows physical, communication, and cognitive skills that are necessary to function. From 2015 to 2017, the subject has shown a gradual improvement from moderate to mild disability in mobility, use of hands, and dizziness. $\mathrm{He}$ has also shown improvement in problem solving falling from a severe to moderate level of impairment (25-75\% of the time this impairment is demonstrated). The abilities items have been the primary focus in physical, occupational and speech therapies.

Adjustment (Neurobehavioral) Index Graph 2: This analysis shows emphasis on psychological symptoms, neurobehavioral management including irritability-agitation-aggression, insight development, and family relationship building. The analysis demonstrates improvement with fatigue (falling from severe to moderate), and reduced symptom sensitivity (falling from severe to moderate impairment). He continues with severe neurobehavioral impairment characterized by irritabilityagitation-aggression daily, intermittent but daily need for behavioral modification techniques, intermittent law enforcement assistance, and

Table 3. Outcome comparison of TBI and mixed chronic groups.

\begin{tabular}{|c|c|c|c|c|c|c|}
\hline & \multicolumn{3}{|c|}{ Chronic TBI Group } & \multicolumn{3}{|c|}{ Chronic Mixed Diagnosis Group } \\
\hline & Abilities & Adjustment & Participation & Abilities & Adjustment & Participation \\
\hline $\begin{array}{l}\text { Admission } \\
\text { (SD) }\end{array}$ & $\begin{array}{l}54.43 \\
(8.73)\end{array}$ & $\begin{array}{l}56.60 \\
(6.22)\end{array}$ & $\begin{array}{l}52.73 \\
(7.01)\end{array}$ & $\begin{array}{l}49.05 \\
(7.92)\end{array}$ & $\begin{array}{l}56.66 \\
(7.16)\end{array}$ & $\begin{array}{l}54.05 \\
(8.22)\end{array}$ \\
\hline $\begin{array}{l}\text { Discharge } \\
\text { (SD) }\end{array}$ & $\begin{array}{l}47.70 \\
(8.38)\end{array}$ & $\begin{array}{l}51.23 \\
(8.58)\end{array}$ & $\begin{array}{l}46.17 \\
(6.51)\end{array}$ & $\begin{array}{l}45.80 \\
(7.06)\end{array}$ & $\begin{array}{l}52.29 \\
(8.56)\end{array}$ & $\begin{array}{l}49.76 \\
(7.46)\end{array}$ \\
\hline Differences & 4.73 & 5.37 & 6.56 & 3.25 & 4.37 & 4.29 \\
\hline
\end{tabular}

2017 MPAI-4 Case Performance comparison with Mixed Group Abilities Items

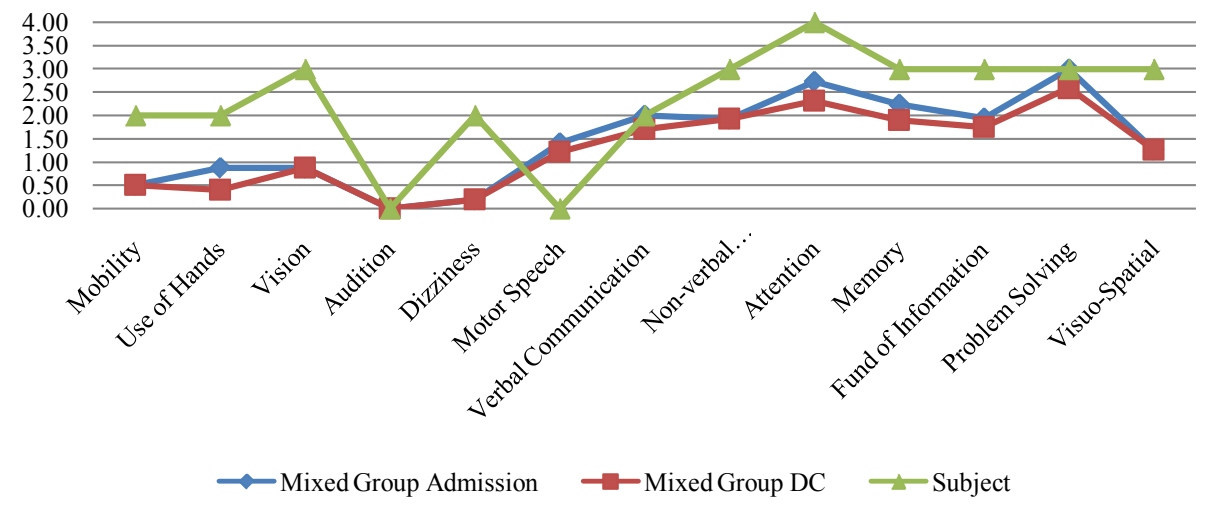

Graph 1. Abilities Index. 
2017 MPAI-4 Case Performance comparison with Mixed Group Neurobehavioral Items

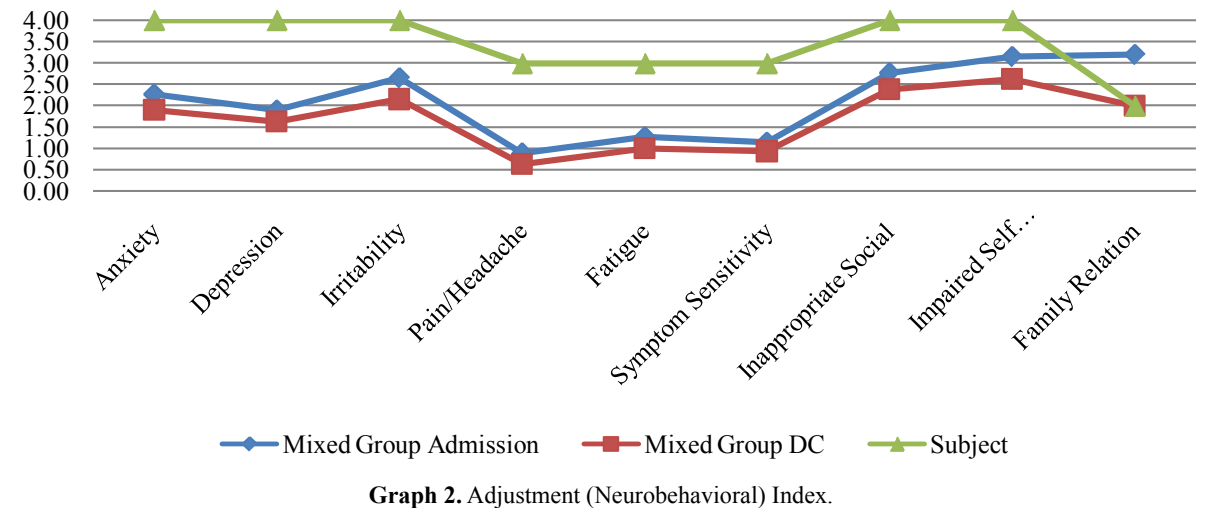

2017 MPAI-4 Case Performance comparison with Mixed Group Participation Items

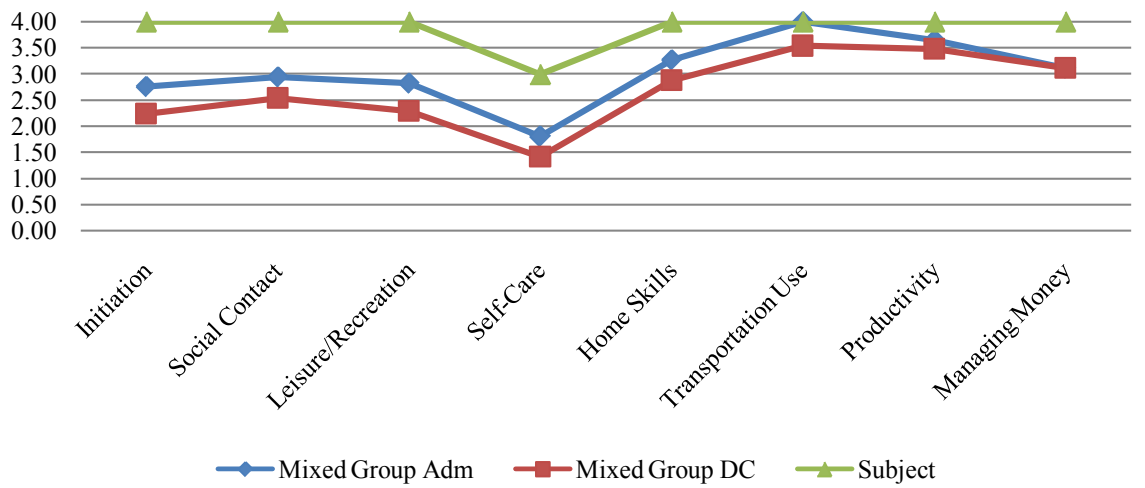

Graph 3. Societal Participation Index.

consistent staff redirection. The physicians monitor the medication types and doses weekly and review the behavioral data to detect small gains achieved over time. With structured and consistent programming, it is reasonable to expect a slow rate of change. Without this level of intervention, one might expect an exacerbation of symptoms with increased disability. The family has been adequately involved with the treatment team to support the consistency of programming.

Societal Participation Index Graph 3: This analysis emphasizes instrumental activities of daily living, which are applied skills that include initiation of activities (also includes inhibition), self-care, home skills, social skills, productive activity (e.g., work, school, volunteering), and money management. The primary skill with demonstrated improvement was self-care. He continues needing cues and redirection up to $75 \%$ of the time, especially for hygiene, but he is making gradual improvement in this level of skill building. This is a necessary skill to improve as part of the overall rehabilitation process and has been demonstrated to be a predictor of successful outcome at the time of discharge [11].

\section{Discussion}

The aim in treatment of craniopharyngioma is long-term survival and disease control with preservation of quality of life through minimizing tumor and treatment-related morbidity. However, this is challenging given the close proximity of these tumors to vital brain structures [4]. Although a benign tumor, it is aggressive due to the nature of how its growth pattern impacts pituitary function, resulting in a mixed presentation of medical, neurological and neurobehavioral sequelae. As such, post-operative treatment tends to be quite difficult and seemingly resistant to intervention. The treatment resistance tends to be related to a combination of fluctuations in medication, social milieu, family and societal support, and environmental challenges.

This case presented provides a few considerations when treating this type of neurological disorder. First, treatment for this type of tumor goes beyond the surgical excision. In addition, treatment may require lifetime intervention at all stages of development depending upon the residual deficits that may occur, especially if the individual is diagnosed within the first incidence peak (ranging from age 5-14). The research is clear in demonstrating that diagnosis in childhood leads to more limited outcome with impairment in social and occupational functioning for the lifetime. Child and adult onset craniopharyngioma survivors were 4.5 to 8 times more likely to live with their parents in adulthood than a control sample. They were also less likely to have a significant other, less likely to have children, with at least some reduction in educational and vocational achievement [12]. Greater difficulties with education and vocational achievement were likely with childhood onset. With support, some form of productive educational and vocational pursuits may be possible, depending upon post-surgical recovery of function. This case has had similar results to the research noted.

Second, due to the complexity of the outcome, intervention has to 
be multifaceted and incorporate many medical specialists including specialized rehabilitation programming with a behavioral emphasis. This treatment must be gradual for behavioral learning to occur, with multiple learning trials that include reinforcement and extinction procedures.

Third, this is a post-surgical condition that is not easily managed. Fluctuations in medical, neurological, and neurobehavioral status must be anticipated throughout the lifetime of the individual with this condition. The case report provides a detailed analysis of many of the complications stemming from this neurological impairment. Although this case demonstrated severe impairment, steady rehabilitation assistance was able to show a gradual change in the level of disability.

\section{Funding information}

Neuro Restorative Research Institute provided financial support for completion of this project.

\section{Competing interest}

There are no conflicts of interest for any of the authors.

\section{References}

1. Müller HL (2014) Craniopharyngioma. Endocr Rev 35: 513-543.[Crossref]

2. Bunin GR, Surawicz TS, Witman PA, Preston-Martin S, Davis F, et al. (1998) The descriptive epidemiology of craniopharyngioma. J Neurosurg 89: 547. [Crossref]

3. Hayhurst C, Williams D, Yousaf J, Richardson D, Pizer B, et al. (2013) Skull base surgery for tumors in children: long-term clinical and functional outcome. $J$ Neurosurg Pediatr 11: 496-503.[Crossref]

4. Sands SA, Milner JS, Goldberg J, Mukhi V, Moliterno JA, et al. (2005) Quality of life and behavioral follow-up study of pediatric survivors of craniopharyngioma. $J$ Neurosurg 103: 302-311.[Crossref]

5. Nielsen EH, Feldt-Rasmussen U, Poulsgaard L, Kristensen LØ, Astrup J, et al. (2011) Incidence of craniopharyngioma in Denmark $(\mathrm{n}=189)$ and estimated world incidence of craniopharyngioma in children and adults. J Neurooncol 104: 755-763. [Crossref]

6. Wijnen M, van den Heuvel-Eibrink, MM, Janssen JA, Catsman-Berrevoets CE, Michiels EM, et al. (2017) Very long-term sequelae of craniopharyngioma. Eur $J$ Endocrinol 176: 755-767. [Crossref]

7. Malec JF, Kragness M, Evans RW, Finlay KL, Kent A, et al. (2003) Further psychometric evaluation and revision of the Mayo-Portland Adaptability Inventory in a national sample. J. Head Trauma Rehabil 18: 479-492. [Crossref]

8. Malec JF, Lezak MD (2008) The Mayo-Portland Adaptability Inventory (MPAI-4) for adults, children, and adolescents. 1-84

9. Lewis FD, Horn GJ, Russell R (2017) Impact of chronicity on outcomes following post-hospital residential brain injury rehabilitation: application of multivariate statistics and Rasch Analysis. Open Journal of Statistics 7: 1-10.

10. Horn GJ, Lewis FD (2016) Behavioral dyscontrol following acquired brain injury: Effectiveness of post-hospital neurobehavioral intensive programs. J Neurol Neuromed 1: $29-33$

11. Lewis FD, Horn GJ (2015) Neurologic continuum of care: Evidence-based model of a post-hospital system of care. Neuro Rehabilitation 36: 243-251.[Crossref]

12. Mitra MT, Jonsson P, Akerblad AC, Clayton P, Koltowska-Haggstrom M, et al. (2017) Social, educational and vocational outcomes in patients with childhood-onset and young-adult-onset growth hormone deficiency. Clinical Endocrinology 86: 526-533. [Crossref]

Copyright: (C2017 Horn GJ. This is an open-access article distributed under the terms of the Creative Commons Attribution License, which permits unrestricted use, distribution, and reproduction in any medium, provided the original author and source are credited. 